\title{
TITLE:
}

\section{Hes1 oscillation frequency correlates with activation of neural stem cells(Dissertation_全文)}

AUTHOR(S):

Kaise, Takashi

\section{CITATION:}

Kaise, Takashi. Hes1 oscillation frequency correlates with activation of neural stem cells. 京都大学, 2021, 博士(医科学)

ISSUE DATE:

2021-07-26

URL:

https://doi.org/10.14989/doctor.k23424

\section{RIGHT:}

DOI:10.1016/j.gep.2021.119170 (c) 2021. This manuscript version is made available under the CC-BY-NC-ND 4.0 license

http://creativecommons.org/licenses/by-nc-nd/4.0/ 


\section{Hes1 oscillation frequency correlates with activation of neural stem cells}

Takashi Kaise ${ }^{\mathrm{a}, \mathrm{b}}$ and Ryoichiro Kageyama, $\mathrm{a}^{\mathrm{a}, \mathrm{b}, \mathrm{c}}$

a Institute for Frontier Life and Medical Sciences, Kyoto University, Kyoto 606-8507, Japan

${ }^{\mathrm{b}}$ Kyoto University Graduate School of Medicine, Kyoto 606-8507, Japan

c Institute for Integrated Cell-Material Sciences, Kyoto University, Kyoto 606-8501, Japan

Correspondence to:

Ryoichiro Kageyama

Institute for Frontier Life and Medical Sciences

Kyoto University

Shogoin-Kawahara, Sakyo-ku

Kyoto 606-8507, Japan

E-mail: rkageyam@infront.kyoto-u.ac.jp

Keywords: Hes1; neural stem cell; Notch intracellular domain; oscillation; quiescence 


\begin{abstract}
Quiescent neural stem cells (NSCs) are occasionally activated to undergo proliferation and subsequent neuronal differentiation. It was previously shown that the transcriptional repressor Hes 1 is involved in both active and quiescent states of NSCs: when Hes1 expression oscillates, it periodically represses the proneural gene Ascll, thereby driving Ascll oscillations, which regulate the active state, while sustained Hes1 expression continuously suppresses Ascl1, promoting quiescence. However, it remains to be analyzed how the transition from quiescent to active states of NSCs is controlled. Here, we found that overexpression of the active form of Notch1 significantly activates NSCs in both in-vitro and in-vivo conditions and that its levels are proportional to NSC activation. The active form of Notch1 induces a burst of Hes1 oscillations in quiescent NSCs, and the frequency of Hes 1 oscillations, rather than the Hes1 peak levels, correlates with the efficiency of NSC activation. These results raised the possibility that bursting Hes 1 oscillations could increase the chance of Ascl1 oscillations in quiescent NSCs, suggesting that Notch1-induced Hes1 oscillation is a cue for a transition from quiescent to active states of NSCs.
\end{abstract}

\title{
1. Introduction
}

In the developing nervous system, neural stem cells (NSCs) actively proliferate while giving rise to various types of neurons and glial cells. By contrast, in the adult brain, NSCs are mostly quiescent and occasionally become active to initiate proliferation and subsequent production of new neurons. The detailed mechanism of how active versus quiescent NSCs are controlled remains to be analyzed (Urbán et al., 2019). Previous studies revealed that Notch signaling plays an essential role in maintenance of both active and quiescent NSCs (Urbán et al., 2019; Sueda and Kageyama, 2020). Differentiating neurons express the Notch ligand Delta-like1 (D111) under the control of proneural genes, such as Ascll and Neurog2, and activate Notch signaling in their neighboring cells (Bertrand et al., 2002; Ross et al., 2003; Imayoshi and Kageyama, 2014). Upon activation of Notch signaling, the transmembrane protein Notch is processed at the membrane region, releasing the Notch intracellular domain. This fragment is then transferred into the nucleus, forms a complex with the essential Notch signaling mediator Rbpj, and induces the expression of downstream genes such as Hes 1 and Hes 5 (Artavanis-Tsakonas et al., 1999; Kageyama et al., 2008). Hes 1 and Hes5 repress proneural gene expression, 
inhibiting neuronal differentiation (Kageyama et al., 2008). Thus, differentiating neurons inhibit neighboring cells from neuronal differentiation via Notch signaling, thereby maintaining NSCs, a process called lateral inhibition (Artavanis-Tsakonas et al., 1999; Kageyama et al., 2008). Disruption of Notch signaling by deleting Rbpj impairs lateral inhibition, leading to depletion of both active and quiescent NSCs (Imayoshi et al., 2010; Ehm et al., 2010).

Accumulating evidence indicates that Notch receptors and their downstream Hes 1 expression dynamics play an important role in differential regulation of the two states of NSCs (Urbán et al., 2019; Sueda and Kageyama, 2020). When Notch1 is inactivated, active NSCs are selectively lost while quiescent NSCs are maintained (Basak et al., 2012). By contrast, deletion of Notch2 or Notch3 activates quiescent NSCs, indicating that Notch2 and Notch3 are required for maintenance of quiescent NSCs (Kawai et al., 2017; Engler et al., 2018). Furthermore, inactivation of Hes 1 and Hes 1related genes leads to exhaustion of both active and quiescent NSCs (Sueda et al., 2019). These results suggest that the Notch1-Hes1 pathway regulates active NSCs while the Notch2/Notch3-Hes1 pathway regulates quiescent NSCs. Interestingly, Ascl1 plays a critical role in activation of NSCs: it is expressed by active NSCs but not by quiescent NSCs and, in the absence of Ascll, all NSCs remain quiescent, indicating that Ascll is absolutely required for activation of NSCs (Pastrana et al., 2009; Kim et al., 2011; Andersen et al., 2014; Pilz et al., 2018). Further analysis showed that in active NSCs, Hes 1 expression oscillates and periodically represses Ascll, thereby driving Ascl1 oscillations, which induce an active state, whereas in quiescent NSCs, Hes1 expression is sustained, continuously repressing Ascll expression, which leads to a quiescent state (Shimojo et al., 2008; Imayoshi et al., 2013; Sueda et al., 2019). Thus, it is likely that induction of Hes1 oscillations (and resultant Ascl1 oscillations) is a cue for transition from quiescent to active states of NSCs, but the detailed mechanism for this transition is still unknown.

Here, to understand the mechanism for transition from quiescent to active states of NSCs, we examined the activity of Notch1 intracellular domain (NICD), an active form of Notch1, in in-vitro and in-vivo NSCs and monitored Hes1 expression dynamics in cultured NSCs by using a live imaging system. We found that NICD overexpression induces bursts of Hes1 oscillations in quiescent NSCs and that the frequency of Hes1 oscillations correlates with activation efficiency of NSCs. 


\section{Results}

2.1. Activation of cultured quiescent NSCs by Notch1

To activate Notch1 signaling at various levels, we induced the expression of NICD as well as mCherry under the control of promoters of Hes1 (Hes1-NICD), Hes5 (Hes5NICD), glial fibrillary acidic protein (Gfap-NICD), and elongation factor la (EF-NICD) genes (Fig. 1A). We first examined the activity of each construct on the Notch signaling target gene Hes 1 by luciferase reporter assay with HEK293 cells (Fig. S1A). Hes1 expression was efficiently activated by EF-NICD and Gfap-NICD and slightly by Hes5NICD but not by Hes1-NICD (Fig. S1A). We next generated lentivirus of each construct and assessed the NICD levels in NSCs infected with each lentivirus. This analysis showed that the EF-NICD virus was the most efficient, followed by Gfap-NICD, Hes5-NICD, and Hes1-NICD viruses in decreasing order (Fig. 1B).

To examine whether Notch1 signaling can activate cultured quiescent NSCs, we next quantified EdU incorporation in NSCs infected with each lentivirus. Lentivirus transducing mCherry alone under the control of the Hes 5 promoter was used as a control (Fig. 1A). Only $2.0 \%$ of the control virus-infected NSCs incorporated EdU in a quiescent condition, and infection with Hes1-NICD or Hes5-NICD viruses did not increase EdU incorporation (Fig. 1C). By contrast, EF-NICD virus significantly increased EdU incorporation (4.8\%), while Gfap-NICD virus slightly did so (3.0\%) (Fig. 1C). Among NSCs infected with EF-NICD, EdU-incorporating activated NSCs expressed higher levels of mCherry than EdU ${ }^{-}$non-activated NSCs (Fig. 1D), suggesting that NSCs expressing higher levels of NICD tended to be activated more efficiently. These results indicated that the activation efficiency of cultured NSCs is proportional to the NICD levels.

When the culture condition was changed to a proliferation medium, only EFNICD virus significantly increased the proliferation rate of NSCs at both Day 3 (Fig. 1E) and Day 6 (Fig. 1F). Gfap-NICD virus did not enhance NSC proliferation (Fig. 1E,F), although it increased EdU incorporation (Fig. 1C). This may have been observed because the Gfap promoter activity might have been weaker in the growth condition than in the quiescent condition. These data suggest that there may be a threshold of the NICD levels that can enhance NSC proliferation. 


\subsection{Activation of quiescent NSCs in the adult brain by Notch1}

It was previously reported that the induction of NICD can activate neurogenesis in the adult brain (Breunig et al., 2007). We next examined whether activation efficiency of NSCs is proportional to NICD levels. To this end, each lentivirus was injected into the hippocampal dentate gyrus of 12-week-old adult mice to infect NSCs, which are mostly quiescent. Two weeks after virus infection, activated NSCs $\left(\operatorname{Sox} 2^{+} \mathrm{BrdU}^{+}\right.$) were quantified. We found that formation of activated NSCs was substantially increased by EF-NICD virus (Fig. 2B,B',F) compared to the control (Fig. 2A,F). However, formation of activated NSCs was not increased by Gfap-NICD, Hes1-NICD or Hes5-NICD (Fig. $2 \mathrm{C}-\mathrm{F}$ ). These results agreed well with the in-vitro data of EdU incorporation and NSC proliferation (Fig. 1), suggesting that the higher the NICD level is induced, the more NSCs are activated in the adult brain.

\subsection{Induction of Hes 1 oscillation in cultured quiescent NSCs by NICD}

We next analyzed Hes1 expression patterns in cultured NSCs established from the Luc2Hes 1 reporter mice, in which firefly luciferase (Luc2) cDNA was inserted in-frame into the $5^{\prime}$ region of the Hes 1 gene in a bacterial artificial chromosome (BAC) clone so that the Luc2-Hes1 fusion protein was expressed (Imayoshi et al., 2013). It was previously reported that the reporter expression correlates well to endogenous Hes 1 expression (Imayoshi et al., 2013), and therefore time-lapse imaging of the reporter expression was performed to monitor Hes1 expression patterns. When the control virus was added to cultured quiescent NSCs, Hes1 expression was mostly steady, with only occasional Hes1 pulses (Fig. 3A and movie S1). Hes1-NICD and Hes5-NICD virus infections did not substantially change the Hes1 expression patterns compared to the control (Fig. 3D,E). By contrast, EF-NICD and Gfap-NICD virus infections induced Hes1 oscillations in cultured quiescent NSCs (Fig. 3B,C and movies S2 and S3). Thus, higher levels of NICD induced Hes 1 oscillations more frequently in quiescent NSCs.

We next quantified NICD-induced Hes1 oscillations. The average duration of each Hes1 pulse observed in NSCs infected with Hes1-NICD, Hes5-NICD, Gfap-NICD, and EF-NICD virus was similar to that of the control (Fig. 4A). The peak levels of Hes1 expression were very similar in NSCs infected with Hes5-NICD, Gfap-NICD, and EFNICD virus but lower in those infected with the control or Hes1-NICD virus (Fig. 4A,B). Thus, Hes1 peak levels did not correlate well with induced NICD levels or activation 
efficiency of quiescent NSCs.

We found that Hes1 oscillations occurred more frequently and robustly when higher levels of NICD expression were induced (Fig. 3), and therefore we next quantified the frequency of Hes1 oscillations. We found that the frequency of Hes1 oscillations was the highest when infected with EF-NICD virus, followed by infection with GFAP-NICD and Hes5-NICD virus in this order (Fig. 4C). These results indicated that the higher the NICD levels were induced, the more frequently Hes1 oscillations occurred. Particularly, 6 or more pulses of Hes1 oscillations appeared within a 24-hour period in NSCs infected with EF-NICD virus (Fig. 4D). Because only EF-NICD significantly activated quiescent NSCs (Figs. 1C,E,F and 2B,B',F), these results suggest that the frequency of Hes1 oscillations correlates well with the activation efficiency of quiescent NSCs. In agreement with this notion, among the EF-NICD virus-infected NSCs, mitotic cells exhibited $\sim 24 \%$ higher frequencies of Hes1 oscillations than non-mitotic cells. Furthermore, after infection with EF-NICD virus, the average Hes1 levels during the trough phases were lowered by $\sim 12 \%$ in mitotic NSCs (Fig. S1B) compared to non-mitotic NSCs (see Fig. $3 \mathrm{~B})$. These results raised the possibility that higher frequencies of Hes1 oscillations may increase the chance of lower Hes 1 expression during the trough phase, thereby increasing the chance of activation of quiescent NSCs.

\section{Discussion}

Notch signaling is essential for the maintenance of both active and quiescent NSCs, (Imayoshi et al., 2010; Ehm et al., 2010; Zhang et al., 2019), but the detailed mechanism by which the transition from quiescent to active states is controlled is yet to be analyzed. In active NSCs, Hes1 expression oscillates with a 2-3 hour periodicity by negative feedback (Hirata et al., 2002; Shimojo et al., 2008; Imayoshi et al., 2013), whereas it is higher and sustained in quiescent NSCs (Sueda et al., 2019), suggesting that Hes1 oscillations are important for activation of NSCs. Indeed, induction of high and sustained Hes 1 expression inhibits proliferation of NSCs (Baek et al., 2006). Furthermore, when Hes1 oscillations are dampened, proliferation of NSCs is impaired, leading to microcephaly (Shimojo et al., 2016; Ochi et al., 2020).

Here, we found that high levels of NICD can activate quiescent NSCs in both in-vitro and in-vivo conditions. Furthermore, high levels of NICD can induce a burst of Hes 1 oscillations in quiescent NSCs. It was previously shown that Notch ligands such as 
D111 activate Notch signaling in a pulsatile manner, causing transcriptional bursting of downstream gene expression (Nandagopal et al., 2018; Falo-Sanjuan et al., 2019; Lee et al., 2019). In addition, the downstream effector Hes1 can repress its own expression by binding to its own promoter, thereby autonomously initiating oscillatory expression (Hirata et al., 2002). The bursting of Hes1 oscillations occurred more frequently in NSCs when higher levels of NICD were induced, suggesting that a certain threshold level of NICD accumulation may be required for inducing Hes1 oscillations.

Our data indicated that the frequency of Hes1 oscillations, rather than the Hes1 peak levels, correlates well with the efficiency of NSC activation. We speculate that if the Hes 1 level during the trough phase becomes sufficiently low, such Hes1 oscillations increase the chance of Ascll oscillations and that such chances would increase when Hes1 oscillations occur more frequently. Indeed, the Hes 1 levels during the trough phases were lower in mitotic NSCs than in non-mitotic NSCs after EF-NICD virus infection. Thus, Notch1-dependent Hes1 oscillations may induce Ascll oscillations, which lead to activation of quiescent NSCs.

\section{Experimental procedures}

\subsection{NSC cultures}

NSC lines were established from the Hes1 reporter mice, in which firefly luciferase (Luc2) cDNA was inserted in-frame into the $5^{\prime}$ region of the Hes 1 gene in a bacterial artificial chromosome (BAC) clone so that the Luc2-Hes1 fusion protein was expressed (Imayoshi et al., 2013). These cells were expanded in proliferation medium $[20 \mathrm{ng} / \mathrm{ml}$ EGF (R\&D Systems), $20 \mathrm{ng} / \mathrm{ml}$ bFGF (Wako), 100 units $/ \mathrm{ml}$ penicillin with $100 \mu \mathrm{g} / \mathrm{ml}$ streptomycin (Nacalai Tesque), N2-MAX supplement (Gibco) in DMEM/F-12 (Gibco)]. To induce quiescence, medium was changed to quiescence medium [40 ng/ml BMP4 (R\&D Systems), $20 \mathrm{ng} / \mathrm{ml} \mathrm{bFGF}, 100 \mathrm{units} / \mathrm{ml}$ penicillin, $100 \mu \mathrm{g} / \mathrm{ml}$ streptomycin, and N-2 MAX media supplement in DMEM/F-12] for 4 days (Martynoga, et al., 2013). Culture dishes and glass-bottom dishes were pre-coated with Matrigel (Corning) at 1.5 $\mu \mathrm{l} / \mathrm{ml}$ final concentration for $30 \mathrm{~min}$. For EdU proliferation assay, $10 \mu \mathrm{M}$ of EdU was added to culture medium 12 hours before fixation.

\subsection{Generation of lentivirus}

The promoters of Hes1, Hes5 and Gfap were cloned from Black6J genome DNA. The 
coding sequence for NICD was subcloned into either CSII-2.7-kb-pHes5-MCS- plasmid, CSII-2.6-kb-pHes1-MCS-plasmid, CSII-2.6-kb-pGFAP-MCS-plasmid, or CSII-1.2-kbpEF-MCS-plasmid for lentivirus production. $84 \mu \mathrm{g}$ of each CSII plasmid, $36.5 \mu \mathrm{g}$ of gagpol plasmid (psPAX2), and $11.7 \mu \mathrm{g}$ of VSV-G plasmid (pMD2.G) were co-transfected into HEK293 cells in five 15 -cm dishes by using $860 \mu \mathrm{l}$ of $1 \mathrm{mg} / \mathrm{ml}$ PolyethylenimineMAX (Polysciences). Cells were cultured in OptiPRO serum-free medium (Gibco) containing $10 \mu \mathrm{M}$ Forskolin, $1 \mathrm{mM}$ sodium pyruvate, and GlutaMax (Thermo), and the medium was harvested 3 days later. $100 \mathrm{ml}$ of the medium was concentrated into $200 \mu \mathrm{l}$ of PBS by centrifugation with $8,000 \mathrm{~g}$ for 12 hours and 13,000g for 4 hours.

\subsection{Luciferase assay}

Hes 1 promoter activity was measured using Hes1-luc, as previously described (Ohtsuka et al., 1999).

\subsection{Lentiviral infection in the adult mouse brain}

For in-vitro analysis, viruses were added to cultured NSCs in growth medium for 4 hours. 24 hours after infection, NSCs were passaged to new culture dishes or glass-bottom dishes. For quiescence induction, growth medium was replaced with quiescent medium 24 hours after massage.

For in-vivo analysis, viruses were delivered stereotactically into the dentate gyrus with the following coordinates: anteroposterior $=-2.15 \mathrm{~mm}$ from bregma; lateral $=$ $\pm 1.75 \mathrm{~mm}$; ventral $=2.2 \mathrm{~mm}$, as previously described (Sueda et al., 2019). Two weeks later, brain sections were immunohistologically examined.

\subsection{Immunological analyses}

For immunocytochemical analysis, cells were fixed with $4 \%$ paraformaldehyde in PBS, permeabilized with $0.1 \%$ Triton X-100 in PBS and blocked in 5\% normal donkey serum / 0.1\% Triton X-100 in PBS. The following antibodies (final dilution and source) were used: rat anti-HAtag (1:1000, Roche 11867423001), goat anti-mCherry (1:1000, SICGEN AB0040-200), and DAPI solution (1:1000, Invitrogen $1 \mathrm{mg} / \mathrm{ml})$. First antibodies were applied overnight at $4^{\circ} \mathrm{C}$ and second antibodies with DAPI were applied for 1 hour at room temperature.

For immunohistochemical analysis of brain tissues, mice were transcardially 
perfused with 4\% paraformaldehyde in PBS. Brains were postfixed with 4\% paraformaldehyde in PBS, cryoprotected by sequential incubation with $15 \%$ and $30 \%$ sucrose in PBS, embedded, and frozen in OCT (Tissue TEK). Serial 20- $\mu \mathrm{m}$ thick frontal frozen sections were cut using cryosection. The following primary antibodies (final dilution and source) were used: rabbit anti-doublecortin (1:500; CST 14802), goat antimCherry (1:500, SICGEN AB0040-200), DAPI solution (1:500, Invitrogen 1mg/ml). First antibodies were applied overnight at $4^{\circ} \mathrm{C}$ and second antibodies with DAPI were applied overnight at $4^{\circ} \mathrm{C}$. Pictures were taken by Dragonfly confocal microscopy (Andor).

\subsection{Bioluminescence imaging of Hes1 expression}

NSCs were plated onto $\phi 27-\mathrm{mm}$ glass-bottom dishes (IWAKI) with $1 \mathrm{mM}$ luciferin (Nacalai Tesque) in quiescence medium. After 4 days of quiescent induction, each dish was placed on the stage of an inverted microscope (Olympus IX81-ZDC) and was maintained at $37^{\circ} \mathrm{C}$. Bioluminescence was measured using Olympus objective lens (UPLFLN $40 \mathrm{O}$ ) and was transmitted directly to a CCD camera (Andor iKon-M), as previously described (Shimojo et al., 2008).

\subsection{Image processing and time-series analysis}

Images were processed by Fiji image analysis software as previously described (Isomura et al., 2017). Stack images were applied to the Spike Noise Filter to remove signals from cosmic rays and then to the Savitzky Golay Temporal Filter to get clear dynamic expression. Fluorescence and bioluminescence of each single cell were quantified by TrackMate (ImageJ plugin). To calculate values near the edges (initial and late $50 \mathrm{~min}$ frames in time-series), data-padding was performed by mirroring. Extreme values were extracted manually and tracks whose recording periods were shorter than 1,200 min were discarded.

\subsection{Statistical analysis}

Statistical analysis was performed using the R software. Statistical differences were examined using one-way ANOVA conducted with Tukey HSD post-hoc test. P values < 0.05 were considered significant. Immunological analyses were performed in triplicate or quadruplicate. 


\section{Acknowledgements}

We thank Akihiro Isomura for technical assistance and Karan Ishii for critical reading. This work was supported by Grant-in-Aid for Scientific Research on Innovative Areas from the Ministry of Education, Culture, Sports, Science, and Technology (MEXT) of Japan (16H06480 to R.K.) and Core Research for Evolutional Science and Technology from the Japan Agency for Medical Research and Development (JP18gm1110002 to R.K.).

\section{References}

Andersen, J., Urbán, N., Achimastou, A., Ito, A., Simic, M., Ullom, K., Martynoga, B., Lebel, M., Göritz, C., Frisén, J., et al. 2014. A transcriptional mechanism integrating inputs from extracellular signals to activate hippocampal stem cells. Neuron 83, 10851097.

Artavanis-Tsakonas, S., Rand, M.D., Lake, R.J. 1999. Notch signaling: cell fate control and signal integration in development. Science 284, 770-776.

Baek, J.H., Hatakeyama, J., Sakamoto, S., Ohtsuka, T., Kageyama, R. 2006. Persistent and high levels of Hes 1 expression regulate boundary formation in the developing central nervous system. Development 133, 2467-2476.

Basak, O., Giachino, C., Fiorini, E., MacDonald, H.R., Taylor, V. 2012. Neurogenic subventricular zone stem/progenitor cells are Notch1-dependent in their active but not quiescent state. J. Neurosci. 32, 5654-5666.

Bertrand, N., Castro, D.S., Guillemot, F. 2002. Proneural genes and the specification of neural cell types. Nat. Rev. Neurosci. 3, 517-530.

Breunig, J.J., Silbereis, J., Vaccarino, F.M., Sestan, N., Rakic, P. 2007. Notch regulates cell fate and dendrite morphology of newborn neurons in the postnatal dentate gyrus. Proc. Natl. Acad. Sci. USA 104, 20558-20563. 
Ehm, O., Göritz, C., Covic, M., Schäffner, I., Schwarz, T.J., Karaca, E., Kempkes, B., Kremmer, E., Pfrieger, F.W., Espinosa, L., et al.. 2010. RВРЈк-dependent signaling is essential for long-term maintenance of neural stem cells in the adult hippocampus. J. Neurosci. 30, 13794-13807.

Engler, A., Rolando, C., Giachino, C., Saotome, I., Erni, A., Brien, C., Zhang, R., ZimberStrobl, U., Radtke, F., Artavanis-Tsakonas, S., Louvi, A., Taylor, V. 2018. Notch2 signaling maintains NSC quiescence in the murine ventricular-subventricular zone. Cell Rep. 22, 992-1002.

Falo-Sanjuan, J., Lammers, N.C., Garcia, H.G., Bray, S.J. 2019. Enhancer proming enables fast and sustained transcriptional responses to Notch signaling. Dev. Cell 50, 411 425.

Hirata, H., Yoshiura, S., Ohtsuka, T., Bessho, Y., Harada, T., Yoshikawa, K., Kageyama, R. 2002. Oscillatory expression of the bHLH factor Hes1 regulated by a negative feedback loop. Science 298, 840-843.

Imayoshi, I., Sakamoto, M., Yamaguchi, M., Mori, K., Kageyama, R. 2010. Essential roles of Notch signaling in maintenance of neural stem cells in the developing and adult brains. J. Neurosci. 30, 3489-3498.

Imayoshi, I., Isomura, A., Harima, Y., Kawaguchi, K., Kori, H., Miyachi, H., Fujiwara, T., Ishidate, F., Kageyama, R. 2013. Oscillatory control of factors determining multipotency and fate in mouse neural progenitors. Science 342, 1203-1208.

Imayoshi, I., Kageyama, R. 2014. bHLH factors in self-renewal, multipotency, and fate choice of neural progenitor cells. Neuron 82, 9-23.

Isomura, A., Ogushi, F., Kori, H., Kageyama, R. 2017. Optogenetic perturbation and bioluminescence imaging to analyze cell-to-cell transfer of oscillatory information. Genes Dev 31, 524-535. 
Kageyama, R., Ohtsuka, T., Shimojo, H., Imayoshi, I. 2008. Dynamic Notch signaling in neural progenitor cells and a revised view of lateral inhibition. Nat. Neurosci., 11, 12471251.

Kawai, H., Kawaguchi, D., Kuebrich, B.D., Kitamoto, T., Yamaguchi, M., Gotoh, Y., Furutachi, S. 2017. Area-specific regulation of quiescent neural stem cells by Notch3 in the adult mouse subependymal zone. J. Neurosci. 37, 11867-11880.

Kim, E.J., Ables, J.L., Dickel, L.K., Eisch, A.J., Johnson, J.E. 2011. Ascl1 (Mash1) defines cells with long-term neurogenic potential in subgranular and subventricular zones in adult mouse brain. PLoS ONE 6, e18472.

Lee, C., Shin, H., Kimble, J. 2019. Dynamics of Notch-dependent transcriptional bursting in its native context. Dev. Cell 50, 426-435.

Martynoga, B., Mateo, J.L., Zhou, B., Andersen, J., Achimastou, A., Urbán, N., van den Berg, D., Georgopoulou, D., Hadjur, S., Wittbrodt, J., et al. 2013. Epigenomic enhancer annotation reveals a key role for NFIX in neural stem cell quiescence. Genes Dev. 27, 1769-1786.

Nandagopal, N., Santat, L.A., LeBon, L., Sprinzak, D., Bronner, M.E., Elowitz, M.B. 2018. Dynamic ligand discrimination in the Notch signaling pathway. Cell 172, 869-880.

Ochi, S., Imaizumi, Y., Shimojo, H., Miyachi, H., Kageyama, R. 2020. Oscillatory expression of Hes 1 regulates cell proliferation and neuronal differentiation in the embryonic brain. Development 147, dev182204.

Ohtsuka, T., Ishibashi, M., Gradwohl, G., Nakanishi, S., Guillemot, F., Kageyama, R. 1999. Hes 1 and Hes 5 as Notch effectors in mammalian neuronal differentiation. EMBO J. 18, 2196-2207.

Pastrana, E., Cheng, L.-C., Doetsch, F. 2009. Simultaneous prospective purification of adult subventricular zone neural stem cells and their progeny. Proc. Natl. Acad. Sci. USA 
$106,6387-6392$.

Pilz, G.-A., Bottes, S., Betizeau, M., Jörg, D.J., Carta, S., Simons, B.D., Helmchen, F., Jessberger, S. 2018. Live imaging of neurogenesis in the adult mouse hippocampus. Science 359, 658-662.

Ross, S.E., Greenberg, M.E., Stiles, C.D. 2003. Basic helix-loop-helix factors in cortical development. Neuron 39, 13-25.

Shimojo, H., Ohtsuka, T., Kageyama, R. 2008. Oscillations in Notch signaling regulate maintenance of neural progenitors. Neuron 58, 52-64.

Shimojo, H., Isomura, A., Ohtsuka, T., Kori, H., Miyachi, H., Kageyama, R. 2016. Oscillatory control of Delta-like1 in cell interactions regulates dynamic gene expression and tissue morphogenesis. Genes Dev 30, 102-116.

Sueda, R., Imayoshi, I., Harima, Y., Kageyama, R. 2019. High Hes1 expression and resultant Ascl1 suppression regulate quiescent versus active neural stem cells in the adult mouse brain. Genes Dev. 33, 511-523.

Sueda, R., Kageyama, R. 2020. Regulation of active and quiescent somatic stem cells by Notch signaling. Dev. Growth Diff. 62, 59-66.

Urbán, N., Blomfield, I.M., Guillemot, F. 2019. Quiescence of adult mammalian neural stem cells: a highly regulated rest. Neuron 104, 834-848. 
Fig.1

A

\section{Lentivirus vector}

Control: Hes5 promoter mCherry-3NLS

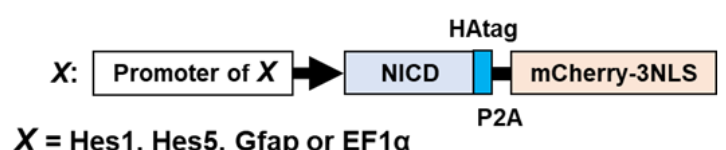

C

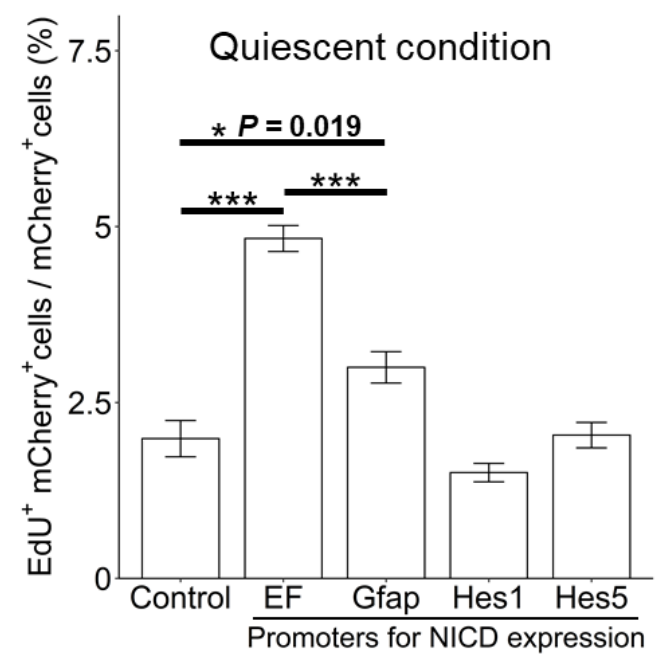

D

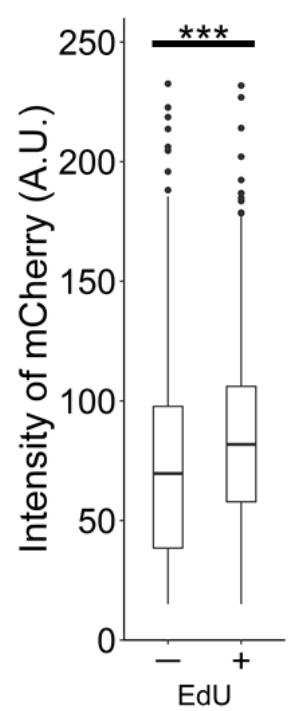

B

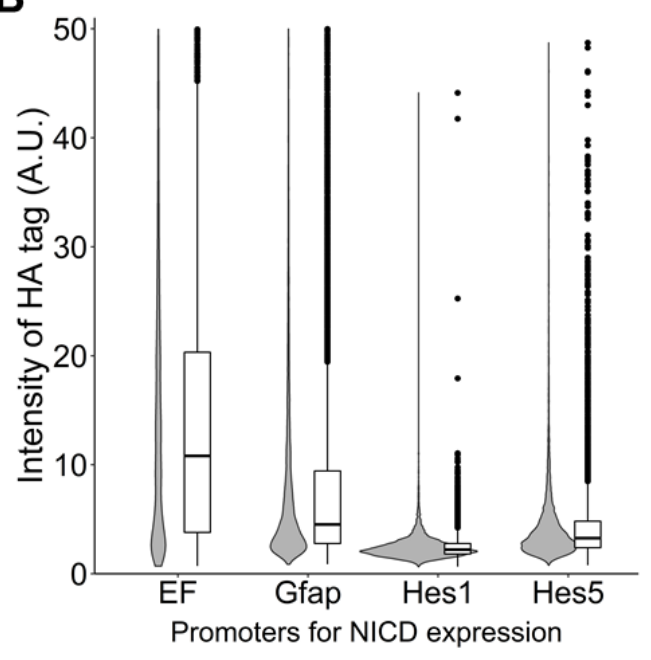

E

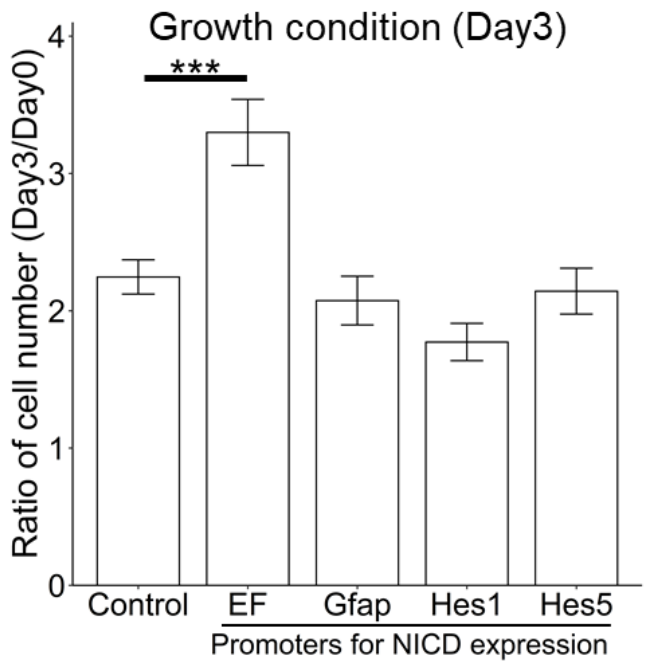

F Growth condition (Day6)

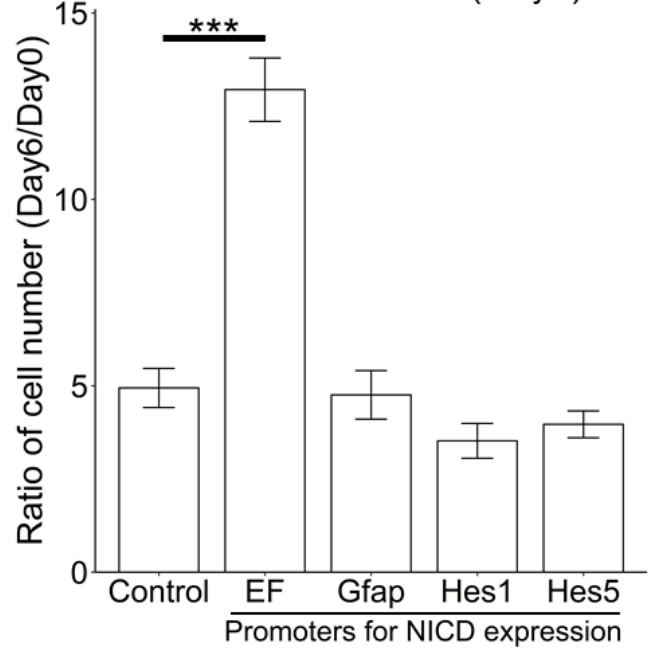


Figure 1. Induction of NICD in cultured NSCs. (A) Schematic structures of lentiviruses. (B) Quantification of induced NICD levels in quiescent NSCs infected with each lentivirus ( $n=4)$. (C) Quantification of EdU incorporation (with SEM) in NSCs infected with each lentivirus $(\mathrm{mCherry})^{+}$, which were maintained in a quiescent medium $(n=4)$. (D) Quantification of mCherry expression levels in EdU ${ }^{-}$non-activated and $\mathrm{EdU}^{+}$ activated NSCs infected with EF-NICD virus. (E,F) Quantification of the number of NSCs infected with each lentivirus, which were maintained in a growth medium. Ratios of the cell numbers on Day 3 (E) and Day 6 (F) relative to those on Day 0 were calculated $(\mathrm{n}=4)$. Each value represents the mean \pm SEM. One-way ANOVA with Tukey HSD post hoc test was conducted. $* \mathrm{P}<0.05, * * * \mathrm{P}<0.001$. 


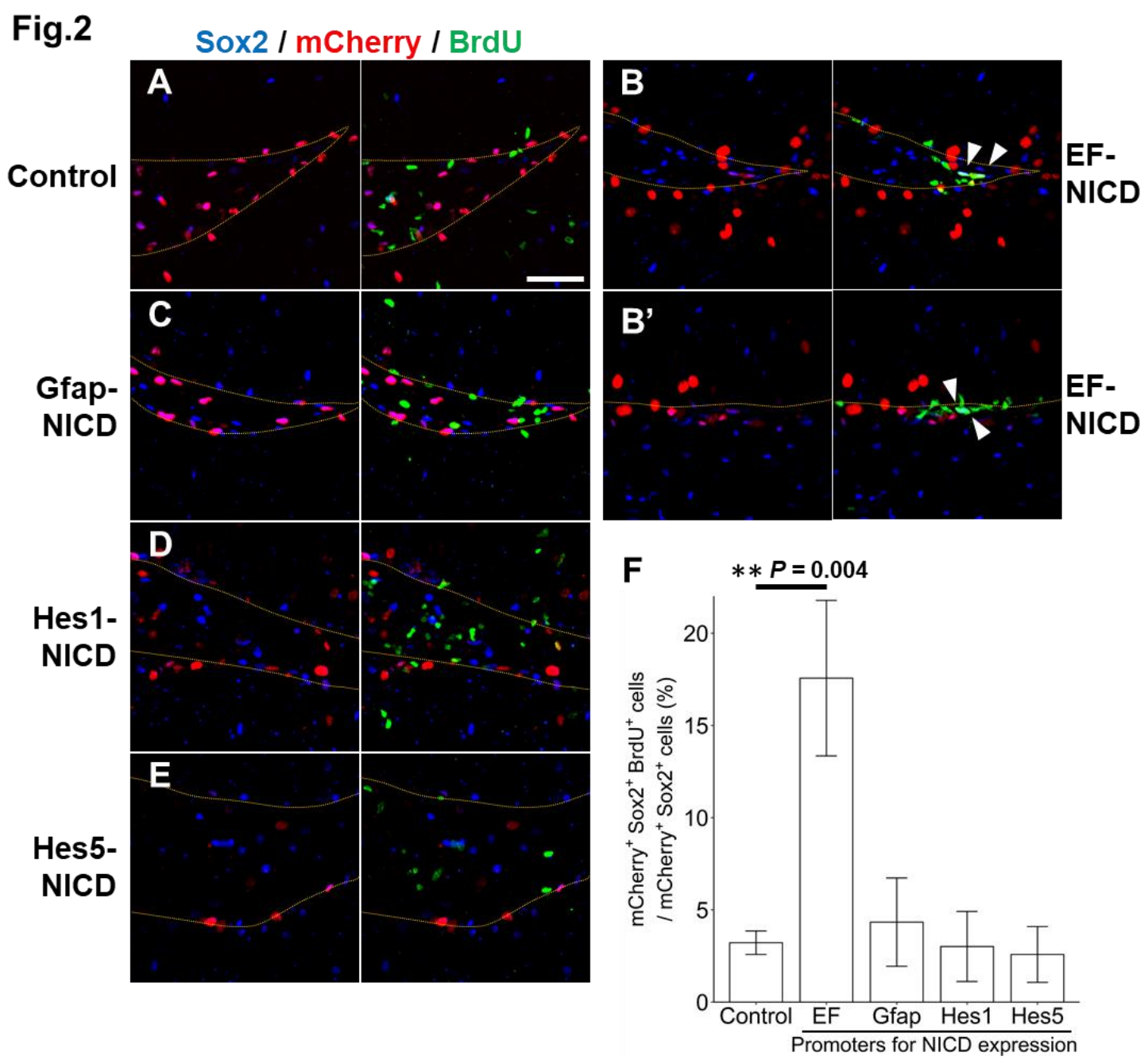

Figure 2. Induction of NICD in the adult hippocampal dentate gyrus. (A-E) The control (A), EF-NICD (B,B'), Gfap-NICD (C), Hes1-NICD (D), and Hes5-NICD (E) viruses were injected into 12-week-old adult mice, and brain sections were immunohistologically examined two weeks later. BrdU-incorporating virus-infected NSCs $\left(\mathrm{Sox}^{+}{ }^{+} \mathrm{mCherry}{ }^{+}\right.$) are indicated by arrowheads. The boundary between the subgranular zone and granule cell layer is indicated by thin lines. Scale bar: $50 \mu \mathrm{m}$ (A-E). (F) Quantification of activated NSCs $\left(\operatorname{Sox} 2^{+} \mathrm{BrdU}^{+}\right)$among the virus-infected cells $\left(m C h e r r y{ }^{+}\right)$. $(n=3$ or 4$)$. Each value represents the mean \pm SEM. One-way ANOVA with Tukey HSD post hoc test was conducted. $* * \mathrm{P}<0.01$. 


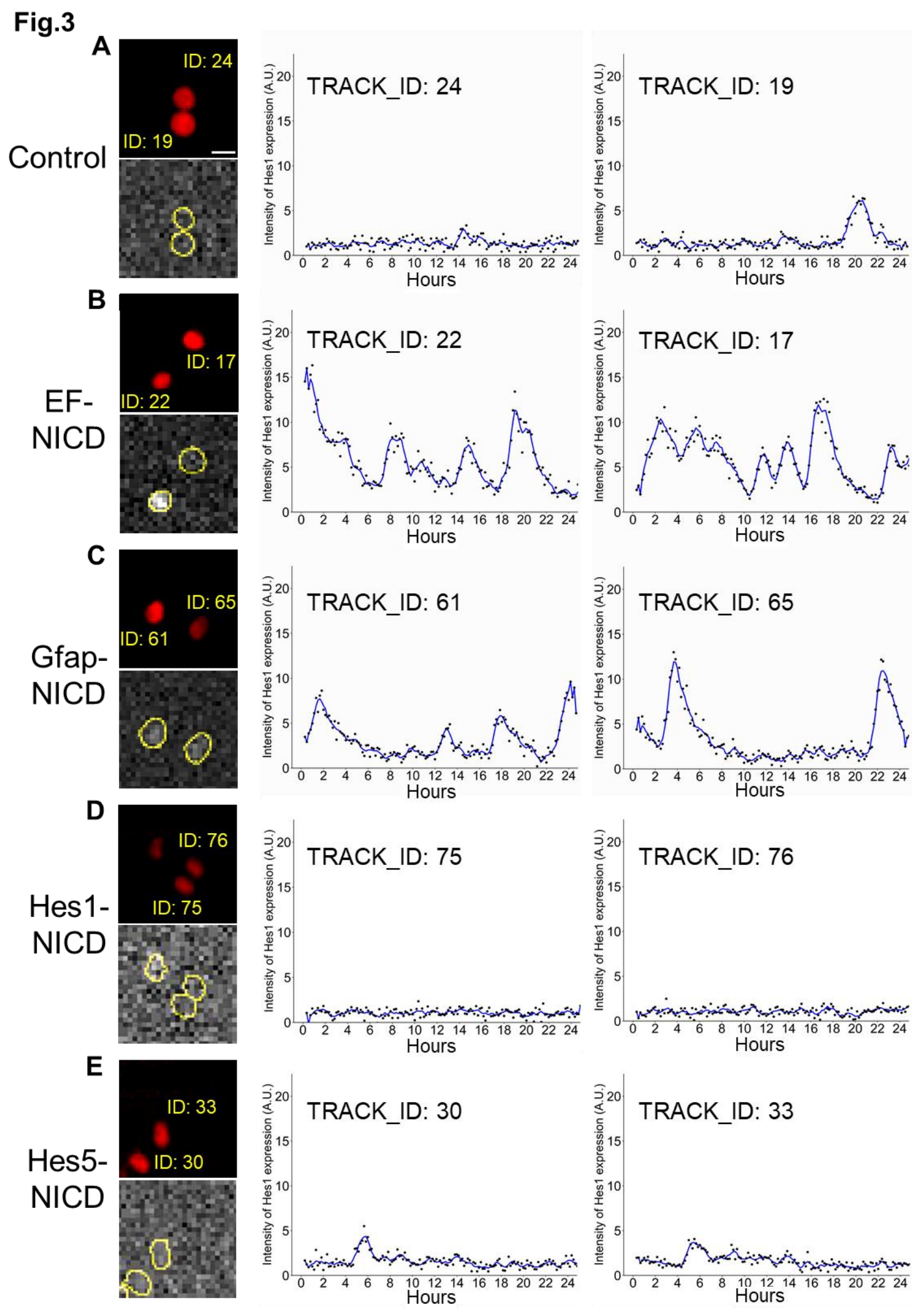


Figure 3. Quantification of bioluminescence imaging of Hes1 expression in cultured NSCs. NSCs carrying the Luc2-Hes1 reporter were infected with the control (A), EFNICD (B), Gfap-NICD (C), Hes1-NICD (D), and Hes5-NICD (E) viruses and were maintained in a quiescent medium. Bioleminescence imaging of virus-infected NSCs (mCherry ${ }^{+}$, circled) was quantified. A.U., arbitrary unit. Scale bars: $10 \mu \mathrm{m}$ (A-E left). 
Fig.4
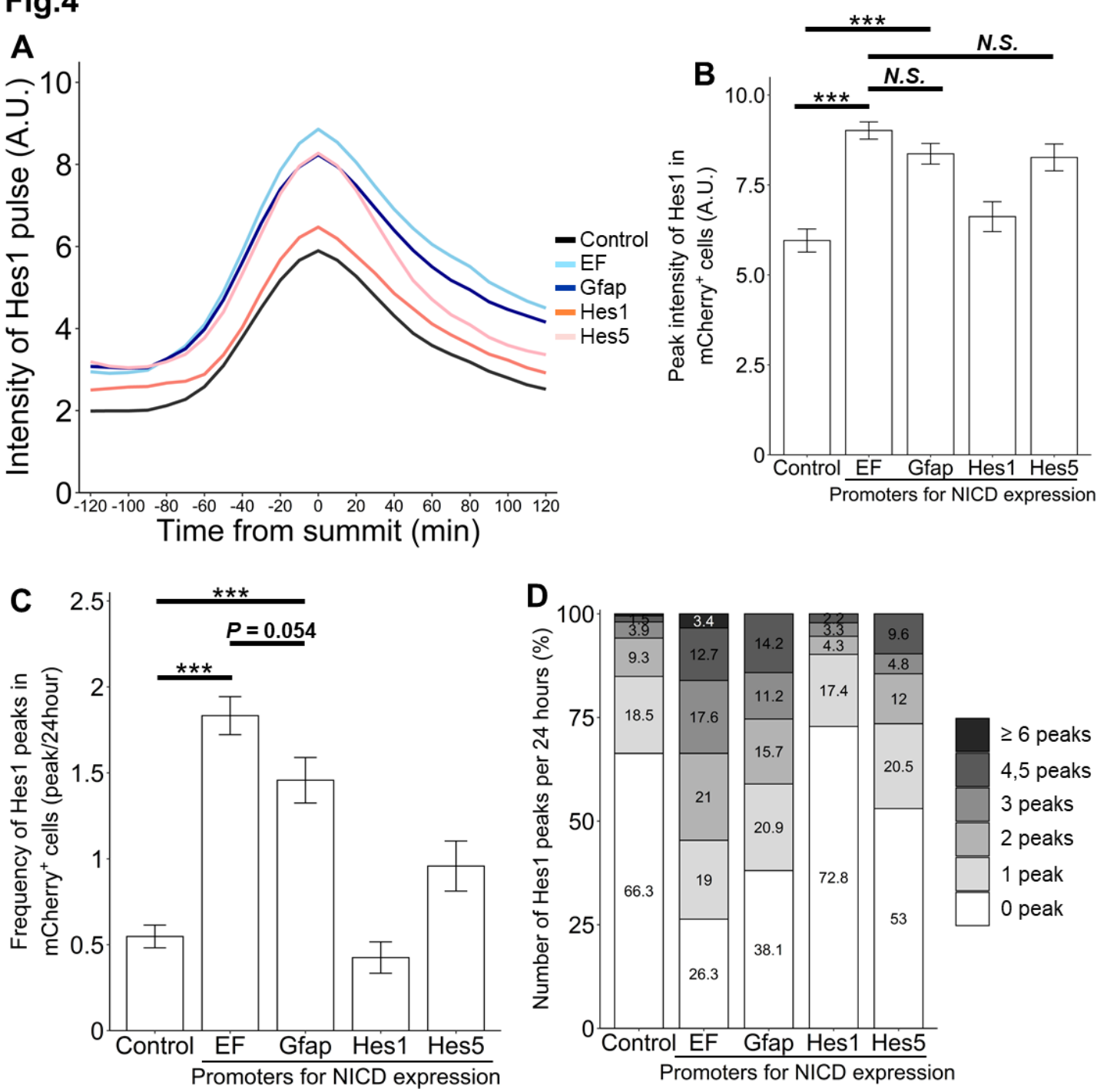

Figure 4. Quantification of Hes1 oscillations in cultured quiescent NSCs induced by NICD. (A) Average pulses of Hes1 oscillations in NSCs infected with the control, EFNICD, Gfap-NICD, Hes1-NICD, and Hes5-NICD viruses. Number of pulses: control=117, EF-NICD=398, Gfap-NICD=195, Hes1-NICD=61, and Hes5-NICD=76. (B) Average peak intensity (with SEM) of Hes1 oscillations. Number of pulses: same as (A). (C) Average frequency (with SEM) of Hes1 oscillations. Number of tracks: control=205, EF-NICD=205, Gfap-NICD=134, Hes1-NICD=137, and Hes5-NICD=83. (D) Quantification of the Hes1 peak numbers per 24 hours in NSCs, which were infected with each virus and were maintained in a quiescent medium. Number of tracks: same as (C). Each value represents the mean \pm SEM. One-way ANOVA with Tukey HSD post hoc test was conducted. $* * * \mathrm{P}<0.001$, N.S. $=$ no statistical significance. 


\section{Supplemental Figure}
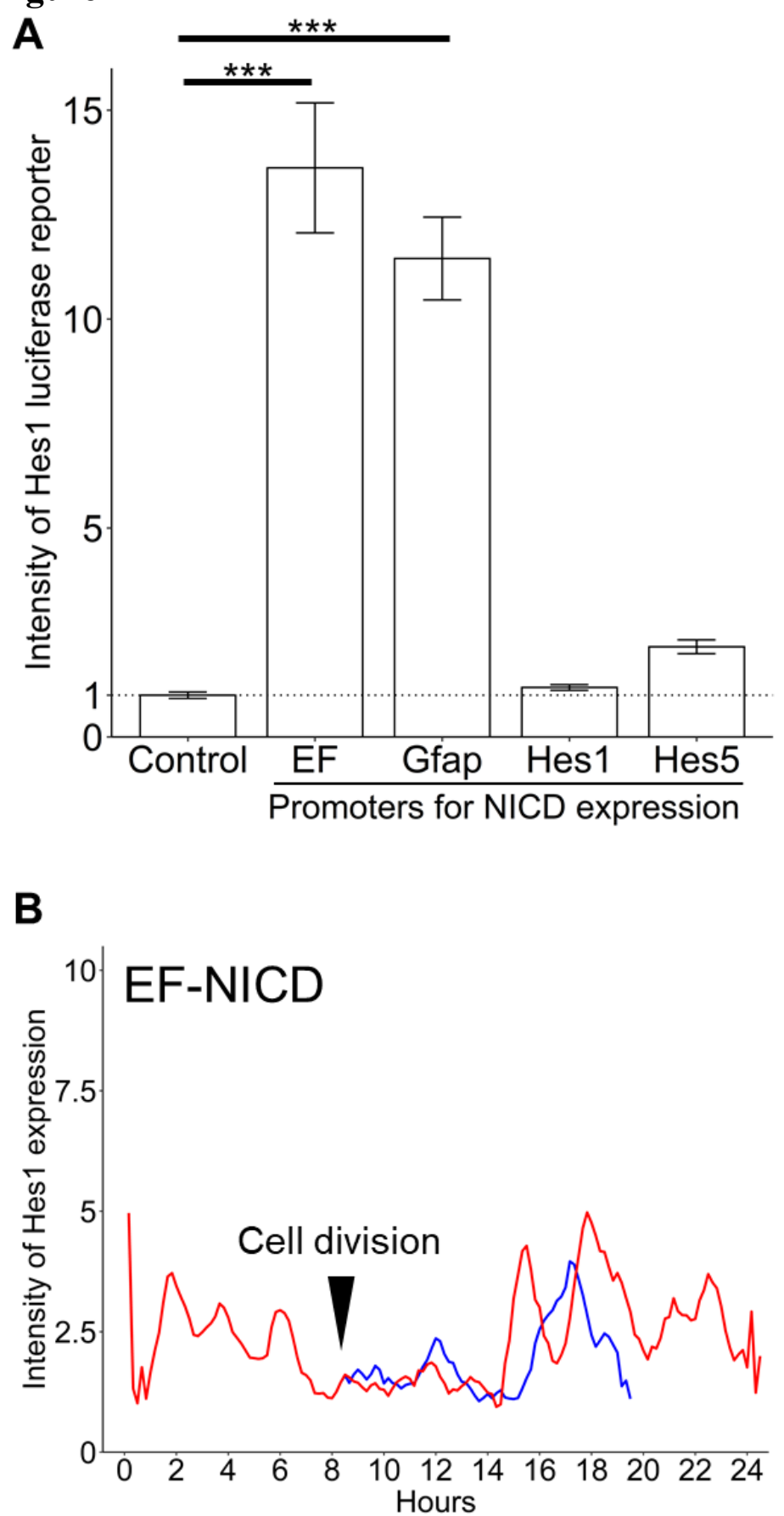

Figure S1. The effect of NICD. (A) Transfection assay of the Hes1 luciferase reporter with the control or NICD expression vectors in HEK293 cells. The relative luciferase activity to the control was quantified $(n=3)$. One-way ANOVA with Tukey HSD post hoc test was conducted. $* * * \mathrm{P}<0.001$. (B) Quantification of bioluminescence imaging of Hes1 expression in cultured NSCs carrying the Luc2-Hes1 reporter that were infected with EF-NICD virus 


\section{Movie legends}

Movie S1. Time-lapse imaging of Hes1 expression in cultured NSCs carrying the Luc2Hes 1 reporter that were infected with the control virus.

Movie S2. Time-lapse imaging of Hes1 expression in cultured NSCs carrying the Luc2Hes 1 reporter that were infected with EF-NICD virus.

Movie S3. Time-lapse imaging of Hes 1 expression in cultured NSCs carrying the Luc2Hes1 reporter that were infected with Gfap-NICD virus. 